\title{
Anesthetic neurotoxicity and the developing brain
}

\author{
Anthony M.-H. Ho MD, Melinda L. Fleming MD, Glenio B. Mizubuti MSc MD
}

Cite as: CMAJ 2017 August 14;189:E1028-9. doi: 10.1503/cmaj.170313

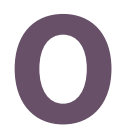

n Dec. 14, 2016, the US Food and Drug Administration warned that anesthetics and sedatives used in children younger than three years of age, or in pregnant women in their third trimester, who were undergoing general anesthesia for more than three hours or used anesthetics repeatedly may affect pediatric or fetal brain development. Children's hospitals across Canada are reportedly advising parents not to delay necessary procedures until their children turn three years old, according to a Mar. 2, 2017 report in The Globe and Mail. Clinicians must be prepared to allay parental concerns over this issue.

The brain undergoes major development in utero and during the first three to four years after birth; it could be vulnerable when exposed to modulators of neuronal activity. Numerous animal studies, several of which are summarized in Appendix 1 (available at www.cmaj.ca/lookup/suppl/doi:10.1503/cmaj $.170313 /-/ D C 1)$, have described neuronal apoptosis and neurocognitive deficits after exposure to anesthetics or sedatives. Many, but not all, studies using routinely collected data - for example, from the Ontario Electronic Medical Record Administrative Data Linked Database, Medicaid and birth registries - have found later neurobehavioural deficits in children who had prolonged or multiple exposures to general anesthesia before two to four years of age.

Fortunately, one brief general anesthetic may not increase the risk. ${ }^{1-3}$ Even if it does, there was only a tiny magnitude of difference between children who had received anesthesia previously and children who were anesthetic-naive (mean age 5.7 years) in the Early Development Instrument score, according to one study. ${ }^{4}$ Moreover, that vulnerability is incurred if the exposure came after 24 months of age, and not before. ${ }^{4} \mathrm{~A}$ Swedish study found a drop of less than $1 \%$ in intelligence quotient (IQ) and school grades, measured during adolescence, associated with exposure to anesthesia at age 37 to 48 months, but not before. ${ }^{5}$ Another study similarly found that exposure to anesthesia before the age of two years was not associated with lower Early Development Instrument performance, but exposure between ages two and four years was. ${ }^{6}$ In these three studies, ${ }^{4-6}$ multiple exposures did not increase vulnerability. Another study showed that six-year-old Dutch children who had been exposed to anesthesia before the age of five years had a mean drop in IQ of 2.1. ${ }^{7}$

\section{KEY POINTS}

- In the human brain, synaptogenesis commences in the last trimester of pregnancy and is complete by the end of the third to fourth year of life. Lasting changes may theoretically occur when this process is disrupted by anesthetics and sedatives.

- Research on animals, including primates, has shown extensive histologic neuronal injury and long-term neurocognitive deterioration after prolonged or multiple exposures to clinical anesthesia.

- Many retrospective human studies have shown neurocognitive deficits in children with multiple exposures to anesthesia before three to four years of age.

- High-level evidence suggests that an exposure to anesthesia for a few hours before three to four years of age causes no long-term harm. The effect of multiple or long exposures is not well elucidated.

- No unnecessary procedures should be performed in children younger than three to four years of age; however, no necessary procedures should be delayed.

Babies born by cesarean delivery are no more likely to have a learning disability in their teen years than those delivered naturally. ${ }^{8}$ Interestingly, babies born by cesarean delivery under spinal or epidural anesthesia have been shown to have lower vulnerability than those delivered by cesarean under general anesthesia and those delivered naturally, possibly due to reduced stress. ${ }^{8}$

The effect on brain development of multiple or prolonged exposures to general anesthesia before the age of three years is the subject of more observational studies. These include the recently completed Mayo Anesthesia Study in Kids (MASK) retrospective-prospective study, which compared neurodevelopmental outcomes in children with no exposures to general anesthesia, those with one exposure, and those with multiple exposures as young children; ${ }^{9}$ the Recognition Memory Study, which addressed prolonged exposure to anesthesia in young children; ${ }^{9}$ and the University of California San Francisco Human Trial, which compared patients with late versus early and long versus short exposure to a volatile anesthetic and anesthesia-naive matched patients, on neuropsychological and comprehension scores at 8 to 11 years of age. ${ }^{9}$ 
However, retrospective observational studies are prone to bias. The impact of surgical illness and hospital admission on development in children can be profound. Otitis and tonsil or adenoid hypertrophy, common pediatric conditions that hinder hearing and sleep and often require anesthesia and surgery, may themselves affect cognitive development. ${ }^{10}$ Databases designed to look at other outcomes may be missing important information about anesthesia, surgery and life experience. Errors in disease coding are common. ${ }^{10}$ Records may not be accurate. Unmeasured confounders may be present despite apparently wellmatched controls.

To settle this issue, data from robust, prospective, ideally experimental studies are needed. Because separating the impact of anesthesia and surgery in children is difficult, human randomized controlled trials (RCTs) must focus on comparing anesthetic techniques that are (thought to be) neurotoxic and non-neurotoxic. In the only RCT to date, 722 children younger than three years underwent inguinal herniorrhaphy with either general anesthesia with sevoflurane (median exposure 54 minutes) or awake spinal or caudal anesthesia. ${ }^{11}$ There was no difference in neurodevelopmental outcomes at two years; the five-year analysis will follow. ${ }^{11}$

In a retrospective-prospective study, children younger than three years who had had general anesthesia for between 20 and 240 minutes showed no differences in memory and learning, motor and processing speed, visuospatial function, attention, executive function language, or behaviour at age 8 to 15 years when matched against anesthesia-naive siblings. ${ }^{12}$ For longer surgical cases, sedation is required even if under regional anesthesia. The T-REX study is a planned RCT that will compare dexmedetomidine-remifentanil (both considered non-neurotoxic and used in combination to optimize intraoperative sedation without prolonging postoperative sedation) versus general anesthesia with sevoflurane after induction with sevoflurane and caudal block in infants for lower abdominal or limb surgery lasting more than two hours. ${ }^{9}$ Neurodevelopmental scores are the presumed primary outcome. For even longer cases, heavy sedation bordering on general anesthesia would be required and may be unsafe. It would be extremely difficult to run an RCT to study the effects of multiple exposures to general anesthesia in children. Future research on anesthetic neurotoxicity from long or multiple exposures to general anesthesia will therefore continue to rely on animal data and clinical studies that have uncontrolled and observational components. Examining the impact of early anesthesia or surgery on youth and young adults would require studies with long timelines.

The signals from animal and observational studies cannot be ignored. However, the observed clinical deficits have been subtle and anesthetic neurotoxicity in children remains unproven. As such, needed procedures must not be delayed because of such concerns. Poor cardiovascular, respiratory and metabolic homeostasis and pain or stress are themselves neurotoxic, and mitigating them must remain the main focus of perioperative management. Meanwhile, clinicians are using regional anesthesia, dexmedetomidine, opioids and muscle relaxants to reduce exposure to potentially more neurotoxic agents. Other potential neurotoxicity-mitigating techniques are being investigated.

Because there is often little choice but to use anesthetics and sedatives, we suggest raising the subject of potential neurotoxicity with parents and clinicians when procedures are wholly elective and can be delayed - an uncommon situation, as pediatric procedures are seldom undertaken lightly. If parents raise the subject, we suggest reassuring them that one brief exposure is likely to be inconsequential, that the effect of prolonged or multiple exposures is unknown, and that neuroplasticity and nurture will probably ensure no long-term sequelae.

\section{References}

1. DiMaggio C, Sun LS, Li G. Early childhood exposure to anesthesia and risk of developmental and behavioral disorders in a sibling birth cohort. Anesth Analg 2011;113:1143-51.

2. Wilder RT, Flick RP, Sprung J, et al. Early exposure to anesthesia and learning disabilities in a population-based birth cohort. Anesthesiology 2009;110:796-804.

3. Sprung J, Flick RP, Katusic SK, et al. Attention-deficit/hyperactivity disorder after early exposure to procedures requiring general anesthesia. Mayo Clin Proc 2012;87:120-9.

4. O'Leary JD, Janus M, Duku E, et al. A population-based study evaluating the association between surgery in early life and child development at primary school entry. Anesthesiology 2016;125:272-9.

5. Glatz P, Sandin RH, Pedersen NL, et al. Association of anesthesia and surgery during childhood with long-term academic performance. JAMA Pediatr 2017;171:e163470.

6. Graham MR, Brownell M, Chateau DG, et al. Neurodevelopmental assessment in kindergarten in children exposed to general anesthesia before the age of 4 years. A retrospective matched cohort study. Anesthesiology 2016;125:667-77.

7. de Heer IJ, Tiemeier H, Hoeks SE, et al. Intelligence quotient scores at the age of 6 years in children anaesthetised before the age of 5 years. Anaesthesia 2017;72:57-62.

8. Sprung J, Flick RP, Wilder RT, et al. Anesthesia for cesarean delivery and learning disabilities in a population-based birth cohort. Anesthesiology 2009;111:302-10.

9. Pinyavat $\mathrm{T}$, Warner DO, Flick RP, et al. Summary of the update session on clinical neurotoxicity studies. J Neurosurg Anesthesiol 2016;28:356-60.

10. Flick RP, Nemergut ME, Christensen K, et al. Anesthetic-related neurotoxicity in the young and outcome measures. The devil is in the details. Anesthesiology 2014;120:1303-5.

11. Davidson AJ, Disma N, de Graaff JC, et al. Neurodevelopmental outcome at 2 years of age after general anaesthesia and awake-regional anaesthesia in infancy (GAS): an international multicentre, randomised controlled trial. Lancet 2016;387:239-50.

12. Sun LS, Li G, Miller TL, et al. Association between a single general anesthesia exposure before age 36 months and neurocognitive outcomes in later childhood. JAMA 2016;315:2312-20.

\section{Competing interests: None declared.}

This article has been peer reviewed

Affiliation: Department of Anesthesiology and Perioperative Medicine, Queen's University, Kingston, Ont.

Correspondence to: Glenio Mizubuti, gleniomizubuti@hotmail.com 